\title{
Uncommon Side Effects of D-Pencillamine- 2 Case Reports
}

\author{
Uma Karjigi*, Dayavathi Ashok \\ Department of Rheumatology, University Hospital of North Durham, Durham, UK \\ Email: ${ }^{*}$ u.karjigi@nhs.net \\ Received 12 December 2013; revised 11 January 2014; accepted 10 February 2014 \\ Copyright (C 2014 by authors and Scientific Research Publishing Inc. \\ This work is licensed under the Creative Commons Attribution International License (CC BY). \\ http://creativecommons.org/licenses/by/4.0/ \\ (c) (i) Open Access
}

\begin{abstract}
D-Penicillamine is one of the diseases modifying medication used to treat Rheumatoid arthritis and Systemic sclerosis. In this article we have described two interesting cases of Dermatomyositis and Bullous pemphigoid secondary to D-Penicillamine. This article emphasizes the fact that D-Penicillamine remains as one of the effective and well-tolerated medications, however patients need close monitoring while on medication, so that uncommon side effects are recognized and treated promptly.
\end{abstract}

Keywords

D-Penicillamine; Dermatomyositis; Bullous Pemphigoid

\section{Introduction}

D-Penicillamine (Dimethylcysteine) has been used successfully for many years in the treatment of Wilson's disease and Cystinuria because of its chelating properties. It is also being used in the treatment of scleroderma and rheumatoid arthritis [1] [2]. In rheumatoid arthritis D-Pencillamine (DP) has been used as either $3^{\text {rd }}$ or $4^{\text {th }}$ line agent, if they have failed to respond or intolerant to conventional therapy. Incidence of side effects with DP amounts to $30 \%$ - $60 \%$, withdrawal rate is $20 \%$ - 30\% [3]. Rare side effects of DP include lupus erythematosus, membranous glomerulopathy, hypersensitivity pneumonitis, Myasthenia Gravis [4], Bullous Pemphigoid and Polymyositis/dermatomyositis (all less than 1\%). DP is associated with development of Dermatopolymyositis (DPM) in $0.2 \%$ to $1.2 \%$ of cases [5].

AIM: In this article we have illustrated two patients who received DP for their underlying conditions and developed uncommon side effects, which were successfully resolved by stopping the drug. Aim of this article is to disseminate the knowledge on the side effects of DP.

*Corresponding author. 


\section{Case Report 1}

A female patient aged seventy-six years was referred in June 2011 with a 6 - 8-month history of pain, swelling and stiffness affecting multiple joints. Examination showed active synovitis of Metacarpophalengeal \& Proximal interphalengeal joints and noted to have thickened skin on dorsum of the fingers, microstomia and telangiectasia. Her ESR was 24 \& CRP of 21, anti-centromere antibody was positive. A diagnosis of Sero negative Rheumatoid Arthritis with limited scleroderma overlap was made. She had great benefit from intra muscular steroid injection. In view of her poor lung function due to Chronic Obstructive Pulmonary Disease Methotrexate was not chosen as treatment option and was started on DP $125 \mathrm{mg}$ daily in September 2011 in view of increasing dose to 250 mg. Joint symptoms improved and normalising inflammatory markers.

In June 2012 she was reviewed urgently as requested by General Practitioner because of erythematous skin lesions with intense itching appeared over her legs initially then spreading to hands, face, and right elbow and back. Felt unwell with muscle aches and stiffness. Examination revealed impressive peri orbital rash (Figure 1) and erythematous lesions over knuckles, back and legs. Her weight was stable and rest of examination was normal including breast examination. Neurological examination showed power 4/5 in proximal and distal muscle groups in all four limbs. Her bloods showed raised Creatinine Kinase 643 and positive ANA 1:640 and negative ENA. We suspected diagnosis of DP induced Dermatomyositis. Penicillamine was stopped immediately and commenced on oral prednisolone $20 \mathrm{mg}$.

She was reviewed by Dermatologist and diagnosed with cutaneous Dermatomyositis on clinical grounds and was commenced on topical steroid ointment.

Rash settled completely with above measures and muscle power returned to normal. She is currently on $5 \mathrm{mg}$ of Prednisolone. Screening for underlying malignancy was negative. At 18-month follow-up she is completely settled.

\section{Case Report 2}

A female patient aged fifty-six years with Rheumatoid Arthritis diagnosed in 2004. She could not continue conventional disease modifying drugs such as Sulphasalazine, Methotrexate and Lefllunomide because of abnormal Liver Function Tests due to non-alcoholic steatohepatitis. She tried Adalimumab followed by Etanercept, unfortunately could not tolerate the side effects and was commenced on DP in February 2011. Dose was gradually increased to $750 \mathrm{mg}$. Her joints remained stable with normalisation of inflammatory markers.

In July 2011 presented with itchy rash and blisters around her ankles. She was reviewed by Dermatologist and had skin biopsy, which showed features consistent with Bullous Pemphigoid. DP was stopped and started on Prednisolone $30 \mathrm{mg}$. Ophthalmologist reviewed her for irritable gritty eyes and diagnosis of early ocular pemphigoid was made.

Continued on prednisolone reducing regime and commenced on Azathioprine as steroid sparing agent which has been very effective in controlling skin, eye and joint symptoms. Follow-up showed all her symptoms resolved.

\section{Discussion}

The mechanisms by which DP causes these side effects are controversial. Two theories have been proposed: a

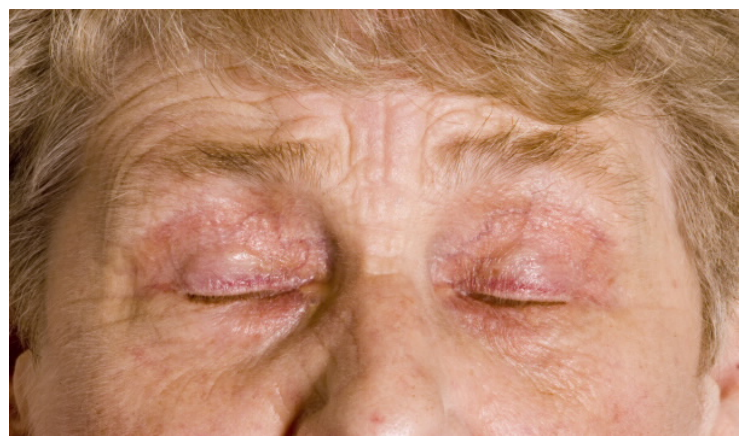

Figure 1. Peri orbital rash. 
modification of the auto antigens due to the presence of the highly reactive thiol group and an interference with the lymphoid cells involved in suppressor or effector lymphocyte cellular co-operation. These complications can be classified into organ specific diseases like myasthenia, polymyositis, thyroiditis and non-organ specific such as sjogren's syndrome and lupus [6]. DP induced PM/DM is less aggressive \& associated with HLA-B18, B35 and DR4 and is immunologically different from idiopathic PM/DM [7].

An analysis of 34 published cases of DP-induced DPM showed development of DPM was not influenced by the dosage or duration of DP therapy. Reported cases were clinically identical with primary DPM but had a different outcome with permanent recovery occurring 1.5 to 6 months after withdrawal of DP as in our patients [5].

It has been suggested that DP therapy might be incidental to the development of polymyositis in patients with Rheumatoid arthritis or that other etiological agents may be present. However, a patient with Wilson's disease treated with DP developed polymyositis, recovered on withdrawal of drug but relapsed on restarting [8].

Rashes are common during treatment with DP, up to $60 \%$ of patients with Wilsons disease can be affected compared to Rheumatoid where lesser dose is used (12\% - 25\%). It was reported that incidence of DP induced Pemphigus is about 7\% and mean duration until onset of Pemphigus is 11 - 13 months [9].

Pemphigus associated with penicillamine therapy was first reported in France, initially in Wilson's disease and later in Rheumatoid arthritis. In these patients prevalence of IgG and IgA antibodies directed against intercellular region tends to be lower even in presence of extensive lesions in contrast with spontaneous pemphigus, where levels correlate with disease activity and used to predict relapses and remissions [10].

There is no evidence to suggest that patients with Rheumatoid arthritis in absence of DP are predisposed to pemphigus.

\section{Conclusion}

D-Penicillamine still remains as an effective treatment for Rheumatoid arthritis especially after failed conventional treatment. However you need to be aware of unusual side effects like in our patients so that it can be treated appropriately and in timely manner. Prompt monitoring is required for patients on DP [11]. Suspension of DP generally leads to resolution of symptoms, but corticosteroids \& immunosuppressant treatment is sometimes required.

\section{References}

[1] Fernandes, L., et al. (1977) Dermatomyositis Complicating Penicillamine Treatment. Annals of the Rheumatic Diseases, 36, 94-95. http://dx.doi.org/10.1136/ard.36.1.94

[2] Lund, H.I. and Nielsen, M. (1983) Penicillamine-Induced Dermatomyositis: A Case History. Scandinavian Journal of Rheumatology, 12, 350-352. http://dx.doi.org/10.3109/03009748309099739

[3] Grasedyck, K. (1988) D-Penicillamine-Side Effects, Pathogenesis and Decreasing the Risks. Zeitschrift fur Rheumatologie, 47, 17-19.

[4] Bucknall, R.C., et al. (1975) Myasthenia Gravis Associated with Penicillamine Treatment for Rheumatoid Arthritis. British Medical Journal, 1, 600-602. http://dx.doi.org/10.1136/bmj.1.5958.600

[5] Kolsi, R., et al. (1992) Dermatopolymyositis Induced by D-Penicillamine in Rheumatoid Polyarthritis. Apropos of 1 Case with Review of the Literature. Revue du Rhumatisme et des Maladies Osteo-articulaires, 59, 341-344.

[6] Meyer, O., et al. (1986) D-Penicillamine: Mechanism of Cellular Action and Induced Autoimmune Diseases. Revue du Rhumatisme et des Maladies Osteo-articulaires, 53, 15-20.

[7] Carroll, G.J., et al. (1987) Penicillamine Induced Polymyositis and Dermatomyositis. The Journal of Rheumatology, 14, 995-1001.

[8] Wright, G.D., et al. (1994) D-Penicillamine Induced Polymyositis Causing Complete Heart Block. Clinical Rheumatology, 13, 80-82. http://dx.doi.org/10.1007/BF02229870

[9] Ho, V.C., et al. (1985) Penicillamine Induced Pemphigus. The Journal of Rheumatology, 12, 583-586.

[10] Marsden, R.A., et al. (1977) Penicillamine-Induced Pemphigus. Proceedings of the Royal Society of Medicine, 70, 103-108.

[11] Doyle, D.R., et al. (1983) Fatal Polymyositis in D-Penicillamine-Treated Rheumatoid Arthritis. Annals of Internal Medicine, 98, 327-330. http://dx.doi.org/10.7326/0003-4819-98-3-327 